\title{
A hybrid time calibration method for EAS ground-based timing arrays
}

\author{
A.Porelli ${ }^{1}$, R.Wischnewski ${ }^{1 *}$, M. Tluczykont ${ }^{2}$ \\ ${ }^{1}$ Deutsches Elektronen Synchrotron (DESY), Zeuthen, Germany \\ ${ }^{2}$ Institut fur Experimentalphysik, University of Hamburg, Germany \\ E-mail: ralf.wischnewski@desy.de
}

\begin{abstract}
In a ground-based Cherenkov EAS timing array the reconstruction of the shower direction is based on the measured photon arrival times at the sensor stations. Relative time offset calibration down to sub-nsec precision is needed for optimal pointing precision. For large arrays, a direct time calibration by artificial light sources introduces a large effort. We discuss a new, hybrid method, that combines information obtained from extended air-showers and a few, dedicated individual station calibrations. We discuss the effect of using exclusively EAS information. The proposed method is particularly useful for large, several squared-km arrays.
\end{abstract}

35th International Cosmic Ray Conference - ICRC2017

10-20 July, 2017

Bexco, Busan, Korea

${ }^{*}$ Speaker. 


\section{Introduction}

In this work we address the problem of the time calibration for a ground-based Cherenkov EAS array. The arrival direction of the EAS primary particle is reconstructed by means of the showerinduced Cherenkov photons space-time informations at the detector level. Thus, the precision of the direction reconstruction depends on how precisely we measure these informations.

The array detector positions are easy to measure and are stable in time. The same is not true for the station time measurements, which depend on detector condition, electronics, cables, stability in time, temperature, etc. The relative time offsets between clocks in different array stations play a crucial role in this sense, affecting the detector angular resolution and most important the absolute pointing precision (systematic mispointing). For a Cherenkov-timing array following the HiSCORE-concept [1] - which is used in this paper for illustration purposes - the required synchronization was shown to be of sub-nanosecond precision [2].

A typical conventional end-to-end calibration of Air-Cherenkov arrays uses a central light source, illuminating all (or many) detectors directly, to perform a "full external array calibration". Dedicated runs with the external LED light source of known position are used to measure and correct the time offsets of the array stations (see e.g. [3]). This aproach, however, becomes difficult when the size of the array (and the number of stations as a consequence) increases up to several $\mathrm{km}^{2} \mathrm{~s}$, together with the need of periodical checks due to the variation of the detector conditions in time. We mention, that another calibration concept makes use of well synchronized light pulsers in each station, to emit known light pulses to all detectors/PMTs to perform regular time offset calibrations (see e.g. AIROBICC [4]). Again - with future array dimension of km-scales, the distribution of precision clocks to all detectors is a challenge by itself, comparable in complexity to the array calibration, thus not allowing a full end-to-end verification. Upcoming reliable and verified clock distribution systems, like discussed in [5], might help to solve this issue.

Another concept for array time calibration is the 'self-calibration' of the full detector array using the collected EAS data [6]. This method avoids the direct experimental calibration of all individual detectors by artificial sources. It is based on two steps: (1) a first minimization of the reconstruction fit residuals, Residual correction, and (2) a final correction, based on additional information that contains directional input (otherwise, no time- or pointing-calibration is possible). Here, the concept of the array's Characteristic Plane ( $\mathrm{CP}$ correction), introduced in [6] plays a role, and is needed to fully correct the mispointing introduced by the non-zero initial station time offsets. One way to obtain the $C P$ correction, as presented in [6] and applied in [7, 8], is based on the assumption of an a priory knowledge of the distribution of the true arrival directions of the detected EAS used for the calibration. However, it is not always easy to justify such an assumption with the required precision (as e.g. discussed in [9]), limiting the application of this self-calibration method. Inhomogenous detector acceptance, while tolerable for the main physics aim, can influence the absolute pointing calibration of the array significantly, and would thus limit the precision of the instrument.

We propose in this paper a new, "hybrid" time calibration method. It is based on a different solution for the CP problem: it combines the detected EAS data with the precise external measurement of the time offsets for a small subset of all array stations. This "hybrid" method does not rely on the knowledge of true directions of the detected EAS (i.e. the complete EAS-acceptance of the 


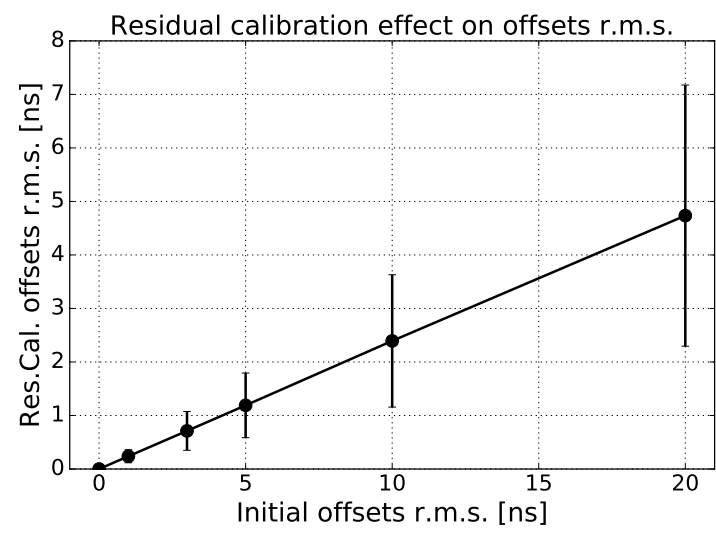

(a)

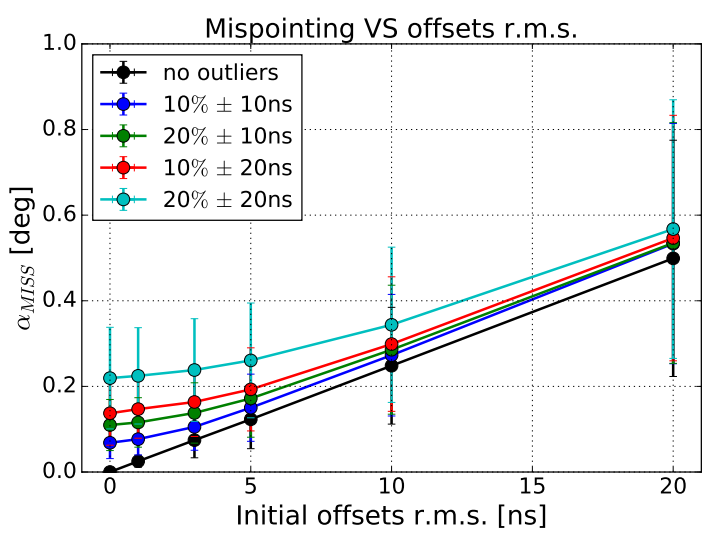

(b)

Figure 1: Calibration result, after applying only the Residual correction: (a) Correlation between the station time offsets r.m.s. before and after applying the Residual correction. (b) Mispointing angle $\alpha_{M I S}$ as function of the initial time offsets r.m.s (from an uniform distribution). A few initial outliers can lead to a big systematic mispointing, even for a good intial guess for most of the time offsets. In this example, $\pm 10 / 20 \mathrm{~ns}$ are added to a different fraction of array stations (10\% or 20\%), resulting in a large pointing error $\alpha_{M I S}$ even for small average initial r.m.s. time offsets.

array), as required by the self-calibration method. At the same time it avoids the difficulties usually encountered for the "full external array calibration", when calibrating a large array with dedicated calibration runs for all stations.

The new hybrid method has been verified by a set of MC simulations as shown in section 3. It has been successfully used to calibrate the TAIGA-HiSCORE detector [1].

\section{Self-calibration of time-offsets: The CP-method}

A detailed mathematical description of how the station offsets affect the direction reconstruction in a ground-based EAS array is presented in [6] and [7]. The CP method is also introduced, together with the solution for the correct estimation and correction of the station time offsets. We summarize the main idea here, which is useful to derive also the hybrid method.

In a ground-based EAS array, for an event $i$, the arrival time $t_{i}$ is measured at each detector unit $j\left(\mathrm{x}_{j}, \mathrm{y}_{j}, z_{j}\right)$. The arrival direction angles, $\theta_{i}$ and $\phi_{i}$, are reconstructed with a least squares fit. If $\Delta t_{j}$ is the typical station time offset of the $\mathrm{j}$-th station, the shower front equation goes like:

$$
c\left(t_{i j}-\Delta t_{j}-t_{0 i}\right)=x_{j} l_{i}+y_{j} m_{i}-z_{j} n_{i}+\omega\left(R_{i}\right)
$$

where $c$ is the speed of light, $l_{i}=\sin \left(\theta_{i}\right) \cos \left(\phi_{i}\right), m_{i}=\sin \left(\theta_{i}\right) \sin \left(\phi_{i}\right)$ and $n_{i}=\cos \left(\theta_{i}\right)$ are the direction vector cosines. $\omega\left(R_{i}\right)$ describes the shower front deviation form the planar solution as a function of the station distance from the shower axis, and $t_{0 i}$ is another fit parameter. The goal of the time calibration is to determine the unknown values of the station time offsets $\Delta t_{j}$.

A common way to correct the times is reducing the residuals with respect to the shower front fit: Residual correction, $\delta_{i}^{\text {res }}$. However, as will be also discussed below, the Residual correction does not guarantee the full correction of the time initial offsets. This is shown in Figure 1, where for 


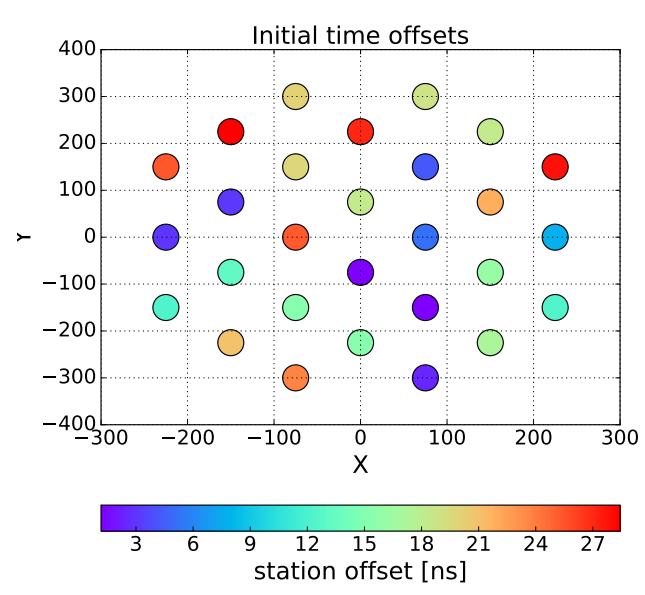

(a)

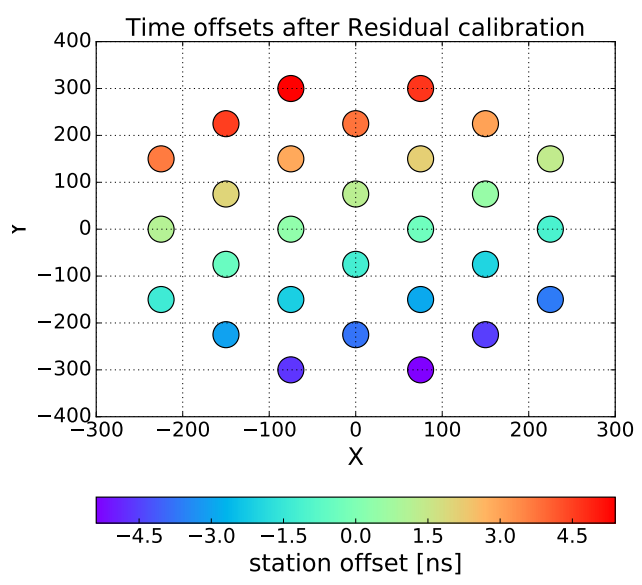

(b)

Figure 2: Example of time offset alignment on the Characteristic Plane (CP) due to the Residual correction, given for the example array (28 HiSCORE stations [1]); the color-code gives the time-offset from perfect calibration. (a) Initial time offset distribution. (b) Time offset distribution after Residual calibration. The color-coded offset pattern defines the resulting array $\mathrm{CP}$.

a gaussian distribution of initial time offsets, after the Residual correction step still about $20 \%$ of the inital time offsets are remaining (Fig.1 left). More importantly, a systematic mispointing is present after the Residual correction (Fig.1 right). In this and the following examples, the HiSCORE array [1] is used.

Giving this observation, it is useful to assume that the calibration process needs to determine two components, i.e. the time offsets calibration $\Delta t_{j}$ is the sum of two terms: the residual and another unknown term. After applying the Residual correction the shower front fit equation becomes:

$$
c\left(t_{i j}-\delta_{j}^{r e s}-t_{0 i}^{\prime}\right)=x_{j} l_{i}^{\prime}+y_{j} m_{i}^{\prime}-z_{j} n_{i}^{\prime}+\omega\left(R_{i}^{\prime}\right)
$$

resulting in the inaccurate direction cosines $l_{i}^{\prime}=\sin \left(\theta_{i}^{\prime}\right) \cos \left(\phi_{i}^{\prime}\right), m_{i}^{\prime}=\sin \left(\theta_{i}^{\prime}\right) \sin \left(\phi_{i}^{\prime}\right)$ and $n_{i}^{\prime}=$ $\cos \left(\theta_{i}^{\prime}\right)$ Combining (2.1) and (2.2), we obtain:

$$
\Delta_{j}=A_{i} \frac{x_{j}}{c}+B_{i} \frac{y_{j}}{c}-C_{i} \frac{z_{j}}{c}+\delta_{j}^{r e s}+\delta_{0 i}+\delta \omega_{i}
$$

where $A_{i}=l_{i}^{\prime}-l_{i}, B_{i}=m_{i}^{\prime}-m_{i}$ and $C_{i}=n_{i}^{\prime}-n_{i}$. The constant $\delta_{0 i}=t_{0 i}^{\prime}-t_{0 i}$ is equal for all the stations. The term $\delta \omega_{i}=\omega\left(R_{i}^{\prime}\right)-\omega\left(R_{i}\right)$ is a small radial deviation from the planar solution.The quantities $A_{i}, B_{i}$ and $C_{i}$ determine the Characteristic Plane (CP), representing the difference between the reconstructed fake plane (FP) and the real plane (RP). The CP of a detector array is defined as the average difference between FPs and RPs of the reconstructed events, i.e. the systematic deviation between FP and RP (pointing accuracy). The array CP is fully determined by the equation:

$$
\Delta t_{j}=A \frac{x_{j}}{c}+B \frac{y_{j}}{c}-C \frac{z_{j}}{c}+<\delta_{j}^{r e s}>+<\delta_{0}>
$$


with the plane cosines defined as follow:

$$
\begin{aligned}
& A=<l^{\prime}>-<l>=\sin \left(\theta_{0}\right) \cos \left(\phi_{0}\right) \\
& B=<m^{\prime}>-<m>=\sin \left(\theta_{0}\right) \sin \left(\phi_{0}\right) \\
& C=<n^{\prime}>-<n>=\cos \left(\theta_{0}\right)
\end{aligned}
$$

and where $\left(\theta_{0}, \phi_{0}\right)$ is the direction that defines the normal vector to the array $\mathrm{CP}$.

From (2.4) we see that, knowing the values of $A, B, C$, and of the residual correction $\left\langle\delta_{j}^{\text {res }}\right\rangle$, the time offset of the $\mathrm{j}$-th station is univocally determined by its coordinate $\left(\mathrm{x}_{j}, \mathrm{y}_{j}, z_{j}\right)$.

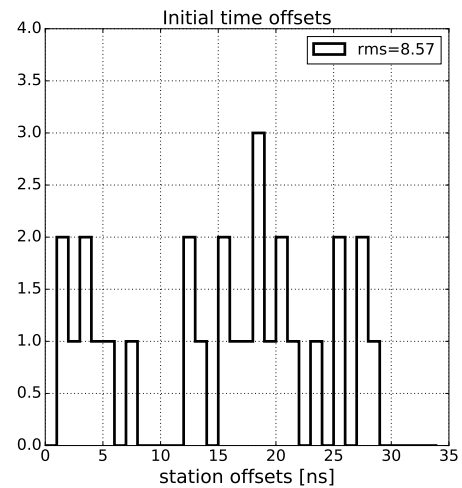

(a)

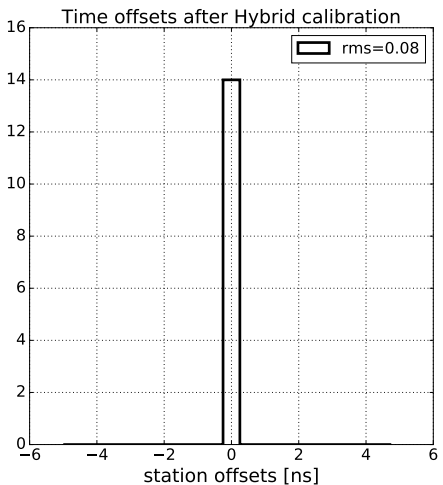

(b)

Figure 3: Example of the Hybrid calibration procedure (MC). (a) Initial time offset distribution (flat). (b) Time offset distribution after the Hybrid calibration: final offsets are $\sim 0 \mathrm{~ns}$ (compare to Fig.2(b)).
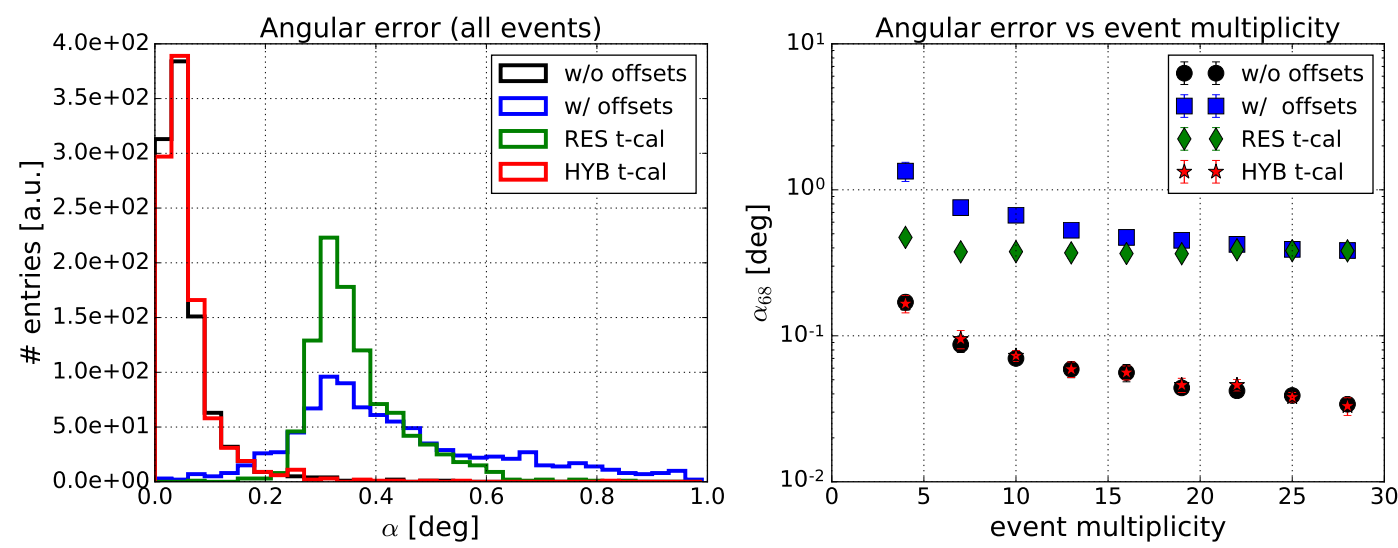

Figure 4: Example of angular error dependence on the station time offsets (simplified MC). Left: Angular error distributions for different sets of station offsets (all events). Right: Angular error $\alpha_{68}$ for different sets of station offsets as function of the event multiplicity. The two Figures show the effect of the different calibration methods: The Residual correction only reduces the error at low multiplicity, but does not correct for the mispointing introduced by the initial time offsets. The Hybrid calibration fully corrects the mispointing, so that the angular error reaches its minimum (i.e. only depends on the intrinsic station time resolution). 


\section{The Hybrid calibration method}

In the previous section we saw, how for a ground-based EAS array a correct determination of the station time offsets can be achieved with the self-calibration method. Two steps are needed: first the Residual correction, second the $C P$ correction. The first one is simply obtained from the reconstruction of the detected events arrival directions. For the second one it is required to know the detected events true arrival direction distribution in order to solve eq. 2.5 and finally eq. 2.4. This needs a precise knowledge of the detector acceptance.

Here, we introduce a new method for time-offset calibration. Another way to solve equation 2.5 is to determine directly the CP parameters, $\theta_{0}, \phi_{0}$ and $t_{0}$, with a linear fit. Rewriting eq. 2.4 as follows,

$$
\delta t_{j}^{C P}=\Delta t_{j}-<\delta_{j}^{r e s}>=A \frac{x_{j}}{c}+B \frac{y_{j}}{c}-C \frac{z_{j}}{c}+<\delta_{0}>
$$

we define the CP plane equation. To solve this 3 parameters system, we need to know the values of $\Delta t_{j}$ (and $\delta t_{j}^{\text {res}}$ ) only for 3 stations. These, as said before, can be obtained in several ways (e.g. manual calibration, external LED light source, etc.). Once the $\mathrm{CP}$ parameters are obtained using this set of "calibration events", it is possible to derive the precise time offsets for all stations of the array.

We call this a "hybrid" time calibration method for ground-based EAS arrays. It combines informations from the EAS (to estimate $<\delta_{j}^{\text {res }}>$ for all the stations, Residual correction) and from external time calibration (for only a few stations, $N \geq 3$, for the $C P$ correction).

\subsection{MC verification}

We validated the "hybrid" calibration method by means of MC simulations. First, we implemented a simple and fast simulation to check the method concept. Then we tested the method using a full EAS simulation, with detector response and EAS event reconstruction. In both simulation tests we used the same set of initial station time offsets, extracted from a uniform distribution between 0 and 30 ns (shown in Figs.2(a), 3(a)). As a detector, a 28 stations array with $\sim 100 \mathrm{~m}$ station spacing is used, as shown in Fig.2 - similar to the HiSCORE geometry [1].

\subsubsection{A simplified simulation}

The first test of the "hybrid" method was performed using a simple simulation (toy-MC), where $10^{4}$ events were generated with azimuth and cos(zenith) uniformly distributed in $\left[0^{\circ}, 360^{\circ}\right)$ and $\left[1, \cos \left(30^{\circ}\right)\right]$ respectively. The arrival time at each station is simulated using a simple plane shower front model, and adding a time jitter of $1 \mathrm{~ns}$ (rms). The event multiplicity is finally obtained randomly choosing $\mathrm{n}$ stations, with $\mathrm{n} \in[4,28]$.

We reconstruct the event directions with a least square fit and collect the residuals for each station. The residual distributions are then fitted with a gaussian function, and the mean values are used to define $\left\langle\delta_{j}^{\text {res }}>\right.$. As expected, applying the Residual correction, the time offsets are aligned on the array CP (Fig.2(b)). In our "hybrid calibration" the CP correction is then obtained as follows: first we assume the 4 central stations as pre-calibrated, and calculate $\delta t_{j}^{C P}$ for each of them; second we fit the 4 station space-time vectors with a plane, obtaining the 3 CP parameters $\left(\theta_{0}^{f i t}, \phi_{0}^{f i t}\right.$ and $\left.t_{0}^{f i t}\right)$. The final step is estimating the station time offset for all the array stations as follows: 


$$
\Delta t_{j}^{H Y B}=<\delta_{j}^{r e s}>+\delta t_{j}^{C P}
$$

Figure 3(b) shows the distribution of the station offsets after this full "hybrid" calibration procedure. The method reduces almost to zero the time offsets, and also removes the reconstruction mispointing as a consequence.

For the angular resolution, the effect of the different calibration steps is shown in Fig.4. The left figure shows the distribution of the angular error $\alpha$ (all events), on the right the angular error $\alpha_{68}$ ( $68 \%$ containment value) as function of the event multiplicity is given. The blue line/squares show the angular error due to the non zero station time offsets (initial offsets). The Residual calibration does not reduce the average mispointing (green versus blue line), it only reduces the error at low multiplicities (green diamonds). It is important to note, that the mispointing is only removed after the next step: applying the full hybrid CP correction (red line/stars). This yields a pointing error like for perfect detector timing calibration (for $\Delta t_{i}=0$, black line/dots).

\subsubsection{Full EAS simulation}

A full EAS MC simulation was performed as a second test. EAS showers were generated using CORSIKA v-6.99 [10], using the IACT package for Cherenkov photon propagation at detection level [11]. We simulated protons of energy between 10 and $5000 \mathrm{TeV}$, with primary particle azimuth uniformly distributed in $\left[0^{\circ}, 360^{\circ}\right)$, and zenith up to $60^{\circ}$. The detector response and the event reconstruction are obtained using the software presented in [2]. We used the true core position to minimize the error in the event direction reconstruction (this simplification has no influence on the conclusions of this work).

The estimation of the station time offsets is performed following the same procedure described in section 3.1.1. In particular, for the first step (Residual correction) only events with station multiplicity $\geq 18$ are selected. The $C P$ correction is obtained assuming the 4 central stations as pre-calibrated. The results of this complete hybrid calibration are shown in Fig.5. The distribution on the left (Fig.5(a)) shows the difference between the initial time offsets and the hybrid calibration correction. The map on the right shows the final station time offsets: all offsets are close to $0 \mathrm{~ns}$ - a radical improvement compared to the Residual correction. In addition, we note a small radial trend is visible due to the presence of the term $\delta \omega_{i}$.

Figure 6 shows the effect of the time offsets on the shower angular reconstruction precision. Again, as discussed in section 3.1.1, we can see how the Residual correction reduces the error only at low multiplicity, but keeps a constant mispointing. Only the full calibration obtained with the hybrid method removes the mispointing completely.

\section{Conclusions}

In ground-based EAS Cherenkov arrays, relative station time offsets need to be calibrated to (sub-) nsec precision - to achieve optimal angular resolution and directional pointing precision. For large area arrays it is difficult to perform an explicit time calibration of the full detector at once (e.g. by external light sources like LED). If frequent checks are required to monitor the detector conditions over time, this becomes an unpractical task. 


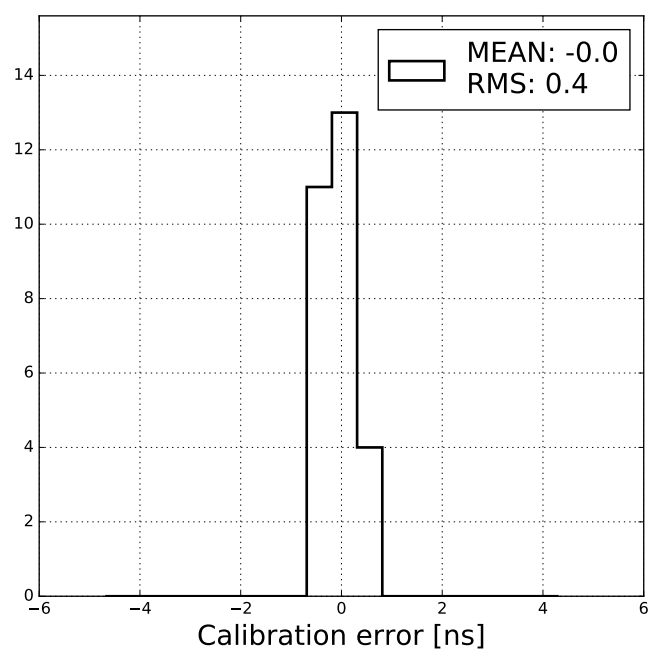

(a)

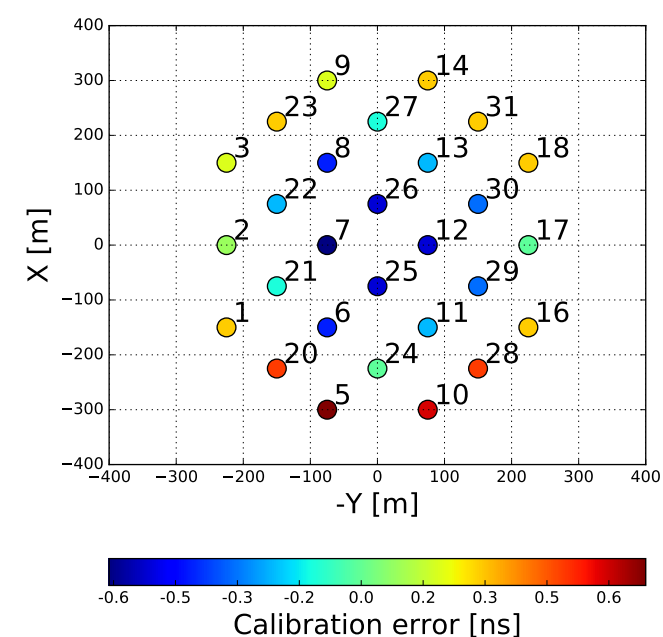

(b)

Figure 5: Hybrid calibration result obtained with full EAS simulation. (a) Final time offsets distribution after the Hybrid calibration. (b) Final time offsets distribution in the array.
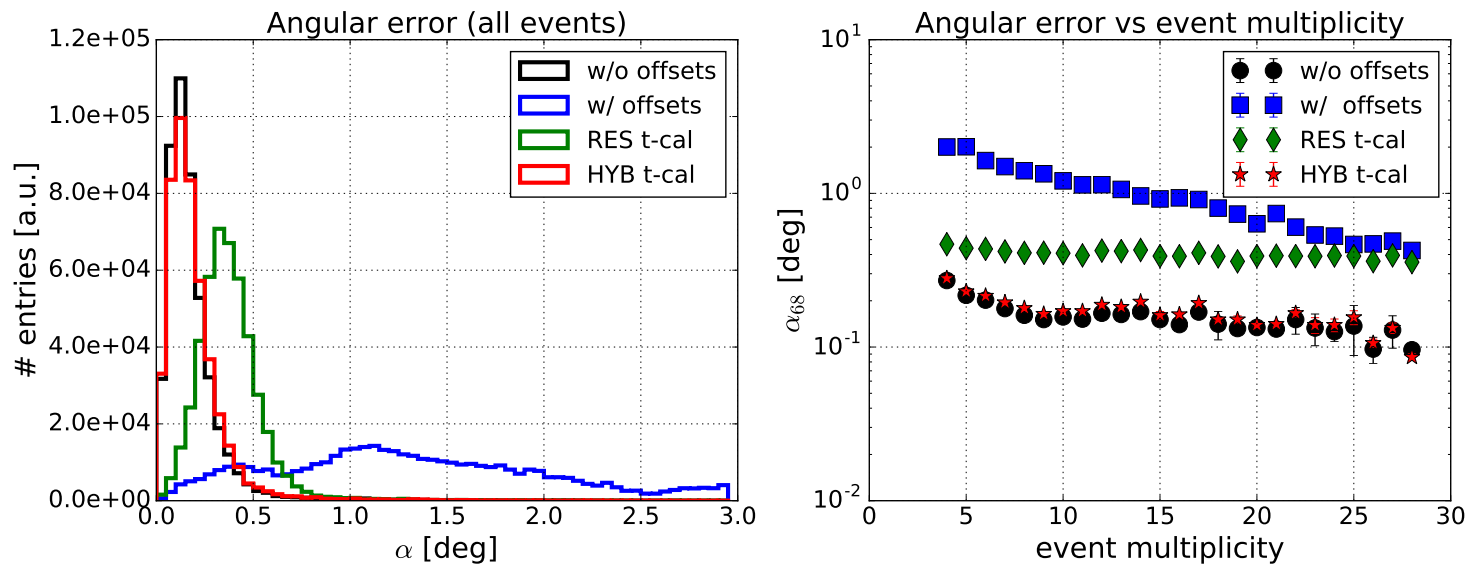

Figure 6: Angular error distribution obtained with the full EAS simulation. Left: Angular error distributions for different sets of station offsets (all events). Right: Angular error $\alpha_{68}$ for different sets of station offsets as function of the event multiplicity. As observed for the toy-MC: The Residual correction only reduces the error at low multiplicity, but does not correct for the mispointing introduced by the initial time offsets. Only the Hybrid calibration fully corrects the mispointing, so that the angular error reaches its minimum (as given by the intrinsic station time resolution).

One solution is given by the self-calibration method, as developed and applied by ARGOYBJ [7]. It allows to calibrate all the array stations at the same time, using the EAS event sample detected during the normal observation time. As discussed in section 2, the time self-calibration (Residual correction $+C P$ correction) allows to precisely determine the station time offsets and the direction mispointing. The disadvantage of the method is that one needs to know a priory the distribution of the arrival direction of the detected events, which is not always possible with the needed accuracy. 
Therefore, we prefer a different approach, and present an alternative solution in this work: the "hybrid" array calibration. The "hybrid method" combines information from both EAS and external time calibration. It formally follows the same mathematical procedure as the self-calibration: The EAS are used for the Residual correction, but the $C P$ correction is obtained fitting directly the CP plane, using the known pre-calibrated time offsets for only few array stations (n-stations $\geq 3$ ). A fast simulation has been used to prove the method, obtaining a reduction of the station time offsets to below $1 \mathrm{~ns}$. A more detailed and realistic full EAS MC simulation confirms these results.

The hybrid time calibration as proposed here has a few advantages: it does not rely on the knowledge of the true distribution of the detected events. Since it requires the external calibration only for a few array stations, it can be performed more frequently and without too much effort, compared to the tedious brut-force approach of external calibration of all detector stations in a large array. Therefore, the proposed hybrid method is a good choice for calibration of upcoming large ground-based EAS Cherenkov arrays.

\section{References}

[1] M. Tluczykont, et al. (TAIGA Collaboration), TAIGA-HiSCORE: results from the first two operation seasons, Proc. 35th ICRC, PoS (ICRC2017) 759; these proceedings

[2] D. Hampf, M. Tluczykont,D. Horns, Nuclear Instruments and Methods in Physics Research, Vol. 712, 137-146, 2013

[3] A. Porelli et al. (TAIGA Collaboration), J. of Phys: Conf. Ser. 632, 012041 (2015)

[4] A. Karle et al., Astropart. Phys, vol. 3, 321-347, (1995)

[5] R. Wischnewski, M Brueckner and A. Porelli, Proc. 34th ICRC, PoS (ICRC2015) 1041

[6] H.H. He, P. Bernardini, A.K. Calabrese Melcarne, and S.Z. Chen, Astropart. Phys., vol. 27,6, 528-532, 2007.

[7] G. Aielli et al. (ARGO-YBJ Collaboration), Astroparticle Physics 30 (2009) 287

[8] P. Bernardini et al. (ARGO-YBJ Collaboration), Proc. 29th ICRC, Pune, 5 (2005) 147-150

[9] B. Bartoli et al. (ARGO-YBJ Collaboration), Phys. Rev. D, 89, 052005, 2014

[10] D. Heck et al. Technical Report FZKA 6019, Forschungszentrum Karlsruhe, 1998.

[11] K. Bernlöhr, Astropart. Phys, vol. 30, 149-158, (2008) 